Apidologie, 1973, 4 (4), 295-315.

\title{
OBSERVATIONS SUR LE DÉVELOPPEMENT D'OSMIA CORNUTA LATR. ET OSMIA RUFA L. (HYMENOPTERA MEGACHILIDAE)
}

\author{
Beobachtungen über die Entwicklung von Osmia cornuta Latr. \\ und Osmia rufa L. (Hymenoptera Megachilidae)
}

\author{
Jean-Noël TASEI \\ avec la collaboration technique de Mile Murielle Picart \\ Laboratoire d'Ethologie et d'Ecologie des insectes, I.N.R.A., \\ 86600 Lusignan
}

\section{SUMMARY}

OBSERVATIONS ON THE DEVELOPMENT OF Osmia cornuta LATR.

AND Osmia rufa L. (Hymenoptera, Megachilidae)

The author carried out investigations into the development of the immature stages and the breaking of imago diapause in $O$. cornuta and $O$. rufa. At $21^{\circ} \mathrm{C}$ the development takes about 19 weeks in $O$. cornuta and 14 weeks in $O$. rufa. The morphological evolution of immature stages is similar in both species. The cocoon's surface is white in 0 . cornuta, brownish in $O$. rufa. In both species the mortality rate is high : $60 \%$ of which $20 \%$ are due to mould and parasites (Melittobia and Cacoxenus).

An exposure to low temperature is necessary for breaking diapause. The precocity and synchronization of adult's emergence at $20^{\circ} \mathrm{C}$ are related to the length of hibernation. 0 . rufa emerge later than $O$. cornuta. Individuals of $O$. cornuta originating from southern areas emerge sooner than those from further north.

\section{RÉSUME}

L'auteur a effectué des observations sur le développement des stades immatures et sur la diapause imaginale d'O. cornuta et $O$. rufa. 
Le développement d'O. cornuta dure environ 19 semaines à la température de $21^{\circ} \mathrm{C}$, celui d'O. rufa 14 semaines. L'évolution morphologique des stades immatures est semblable chez les 2 espèces. Les cocons d' $O$. cornuta sont plus blancs en surface que ceux d'O. rufa. La mortalité chez les deux espèces est très élevée : $60 \%$ dont $20 \%$ sont imputables aux moisissures et aux parasites (Melittobia et Cacoxenus). et

L'hibernation à basse température est nécessaire à l'élimination de la diapause. Plus la phase à basse température est longue, plus les délais de réactivation à $20^{\circ} \mathrm{C}$ sont courts et plus les émergences sont synchronisées. Les délais d'émergence d'O. rufa sont plus longs que ceux d'O. cornuta. Les délais d'émergence sont plus brefs chez les individus d'origine méridionale que ceux d'origine nordique dans le cas d'O. cornuta.

\section{INTRODUCTION}

J'ai précédemment exposé les résultats d'une étude comparative du comportement de nidification chez Osmia cornuta Latr. et Osmia rufa L. (TASEI sous presse). La présente note souligne les différences physiologiques existant entre ces deux espèces et relate dans une première partie, des observations concernant le développement des stades immatures. Dans une deuxième partie, j'étudie l'influence des températures subies lors de l'arrêt de développement imaginal, sur les sorties des adultes.

\section{MATÉRIEL ET MÉTHOdES}

\section{1. - Conditions d'élevage des stades immatures et techniques d'observation du développement}

Les nids sont obtenus dans la région de Lusignan (Vienne) grâce à l'utilisation de piègenichoir du type décrit lors de l'étude sur Osmia coerulescens L. (TASEI, 1972). Les méthodes d'élevage sont les mêmes pour les trois espèces. On protège les portions de cannes de Provence habitées, contre les contaminations de Melittobia acasta Walk. en les enfermant dans des tubes munis de grillage très fin. Une partie de l'élevage est conservée dans un abri de bois placé à un mètre au-dessus du sol. L'autre partie se développe en étuve à $21^{\circ} \mathrm{C}$ environ. L'humidité relative de l'étuve est maintenue dans les limites : 50-80\%. Les nids en étuve sont contrôlés tous les jours. Ceux qui sont conservés à l'extérieur sont visités une fois tous les quinze jours. Le passage d'un stade larvaire à un autre est repéré lors de l'élimination de particules de terre que l'observateur dépose au préalable sur tous les insectes. Cette méthode très pratique a déjà été utilisée par HackwelL (1968) chez Nomia melanderi.

\section{2. - Observations sur l'arrêt de développement imaginal}

En 1966 et 1967 ont lieu les premières expériences. Je récolte à l'automne des adultes d'Osmia cornuta et Osmia rufa provenant soit de vieux nids de Chalicodomes (Megachile pyrenaica Lep.) du Gard et du Vaucluse, soit de nichoirs répartis dans plusieurs départements du Nord et du Midi de la France.

Pendant l'arrêt de développement imaginal divers traitements thermiques sont appliqués aux insectes a fin d'étudier leur in fluence sur les délais d'émergence. Dans une première phase tous les adultes, soit $40 \mathrm{O}$. cornuta et $27 \mathrm{O}$. rufa sont conservés dans leur cocon à une température moyenne de $16-18^{\circ} \mathrm{C}$, du moment où ils sont récoltés jusqu'au $1^{\mathrm{er}}$ décembre. Puis à partir de cette date on répartit les insectes en 6 lots :

4 sont exposés à la température de $0^{\circ} \mathrm{C}$ pendant les temps respectifs de $15,33,61$ et 93 jours

2 sont exposés à la température de $4^{\circ} \mathrm{C}$ pendant les temps de 73 et 110 jours. 
Après ces différentes hibernations ${ }^{1}$ artificielles les lots sont portés en étuve à $20^{\circ} \mathrm{C}$ et on note les délais de réactivation individuels. Le lot témoin ne subit aucune basse température, il demeure à $20^{\circ} \mathrm{C}$ constamment.

En 1970, une expérience complémentaire se déroule dans des conditions entièrement contrôlées : 340 . rufa et 60 . cornuta évoluent aux températures suivantes :

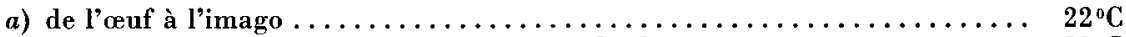

b) Préhibernation de 1,2 ou 3 mois suivant les lots $\ldots \ldots \ldots \ldots \ldots \ldots 20^{\circ} \mathrm{C}$

c) Hibernation de 55 à 150 jours suivant les lots $\ldots \ldots \ldots \ldots \ldots \ldots \ldots \ldots, 3^{\circ} \mathrm{C}$

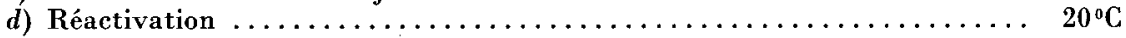

\section{RÉSULTATS}

\section{A. - Le développement des stades immatures}

\section{Le développement dans la nature}

Le tableau 1 donne les résultats de 9 « sondages » effectués tout au long des 5 mois correspondant au développement d'O. cornuta et $O$. rufa.

Il apparaît sur le tableau qu'O. cornuta se développe plus lentement qu'O. rufa. La différence des vitesses de développement est telle que les imagos d'O. rufa apparaissent plus tôt que ceux d'O. cornuta bien que la ponte de la première espèce soit plus tardive. La période comprise entre le filage du cocon et la mue imaginale, dure environ 2 mois chez $O$. cornuta et 1 mois chez $O$. rufa. RAw (1972) a étudié la durée des stades de développement d'O. rufa dans les conditions naturelles en Grande-Bretagne. Il trouve en particulier que le développement de l'œuf à l'adulte demande 103 jours, durée qui est proche de celle indiquée au paragraphe suivant : 98 jours à $21{ }^{\circ} \mathrm{C}$ constant.

\section{Le développement dans les conditions contrôlées}

A la température constante de $21^{\circ} \mathrm{C}$ nous pouvons préciser la durée moyenne de chaque stade (cf. tableau 2).

Étant donné la dispersion des valeurs obtenues, il est bon de consulter le tableau 3 qui indique le nombre de données pour chaque stade et la distribution des fréquences.

J'ai comparé les durées moyennes de chaque stade chez les deux espèces grâce au test $\mathrm{du}$ ( $\mathrm{t}$ ) de Student. Ce test confirme que la durée des stades : ( œuf », ( filage du cocon + prénymphe ), ( nymphe ) est significativement plus longue chez $O$. cornuta que chez $O$. rufa. Seul le stade « larve s'alimentant »

1. On divise l'arrêt d'activité des imagos en 3 phases :

- la préhibernation (de la mue imaginale jusqu'au moment de l'exposition aux basses températures).

- l'hibernation proprement dite à basse température.

- la réactivation à haute température. 


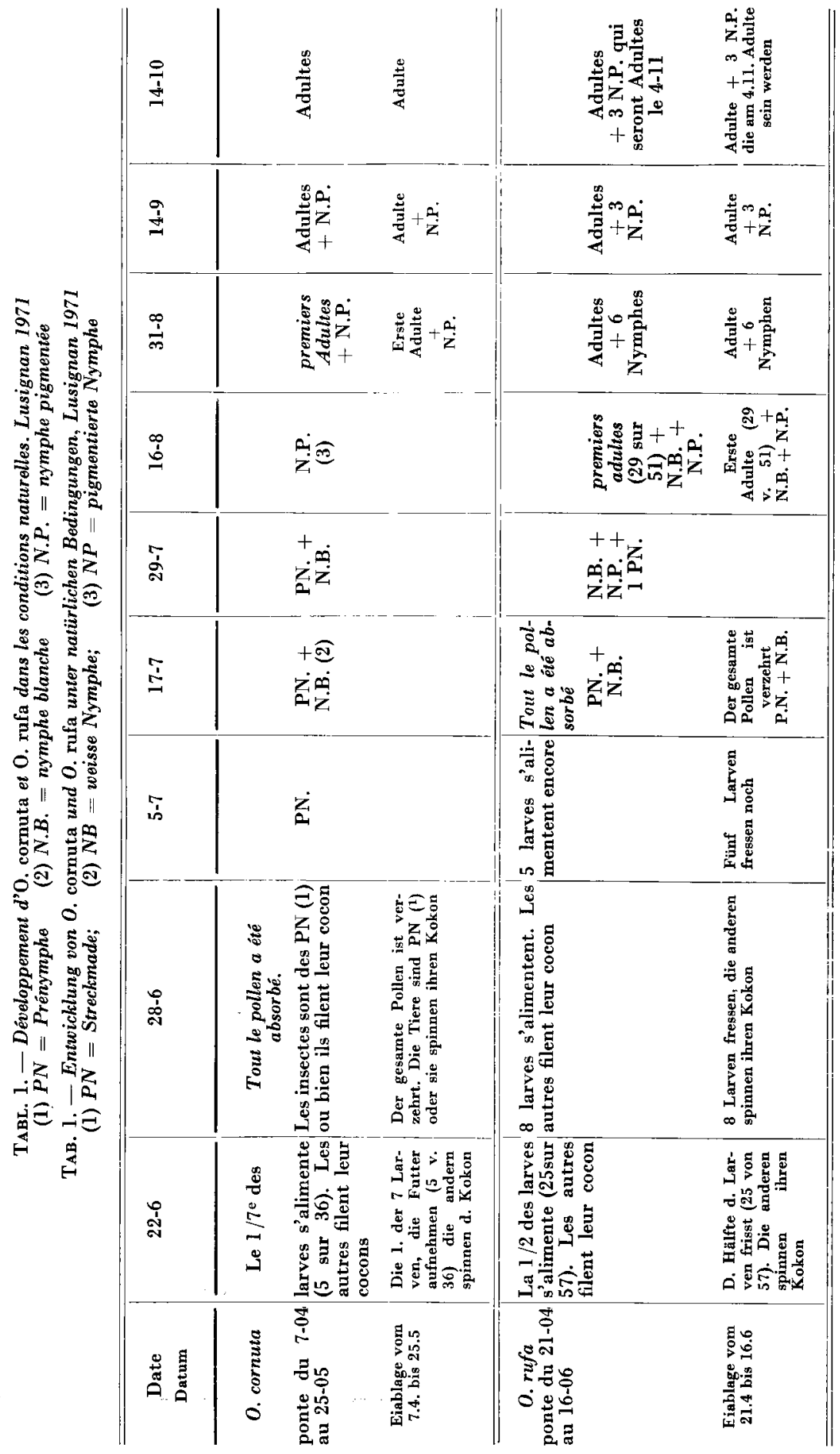


TABL. 2. - Durée des stades de dêveloppement à $21^{\circ} \mathrm{C}$ chez $\mathrm{O}$. cornuta et $\mathrm{O}$. rufa.

(1) Nymphe blanche à cil non pigmenté.

(2) Nymphe blanche à oeil pigmenté.

TAB. 2. - Dauer der Entwicklungsstadien von 0. cornuta u. O. rufa bei $21^{\circ} \mathrm{C}$

(1) weisse Nymphe, Auge nicht gefärbt.

(2) weisse Nymphe, Auge pigmentiert.

\begin{tabular}{|c|c|c|c|c|c|c|c|c|}
\hline & $\begin{array}{c}\text { Stadium } \\
\text { Espèce } \\
\text { Art }\end{array}$ & $\begin{array}{c}\text { Euf } \\
\text { Ei }\end{array}$ & $\begin{array}{l}\text { Larve } \\
\text { s'alimen- } \\
\text { tant } \\
\text { Fressen- } \\
\text { de Larve }\end{array}$ & $\begin{array}{l}\text { Filage } \\
\text { cocon } \\
+ \text { P.N. } \\
\text { Spinnen } \\
+ \text { P.N. }\end{array}$ & $\begin{array}{l}\text { N.B. }{ }^{\left({ }^{1}\right)} \\
\text { o.b. }\end{array}$ & $\begin{array}{c}\text { N.B. }{ }^{(2)} \\
\text { o.p. }\end{array}$ & N.P. & $\begin{array}{c}\text { Dévelop- } \\
\text { pement } \\
\text { total } \\
\text { Gesamtent- } \\
\text { wicklungs- } \\
\text { zeit }\end{array}$ \\
\hline \multirow{2}{*}{$\begin{array}{c}\text { Durée } \\
\text { en jours } \\
\text { Dauer in } \\
\text { Tagen }\end{array}$} & O. cornuta & 8,0 & 24,2 & 64,6 & 4,5 & 18,0 & 16,0 & 135,3 \\
\hline & O. rufa & 7,1 & 27,5 & 29,7 & 3,6 & 16,8 & 12,9 & 97,6 \\
\hline
\end{tabular}

est plus long chez $O$. rufa. Le développement total est significativement plus long chez $O$. cornuta. Le stade “ filage du cocon + prénymphe » dure en moyenne 35 jours de plus chez $O$. cornuta, ce qui confirme les résultats obtenus dans les conditions naturelles (cf. tableau 2). A $21^{\circ} \mathrm{C}$ il faut environ 19 semaines pour qu'un œuf d'O. cornuta donne un imago, alors qu'il en faut seulement 14 dans le cas d'O. rufa. Chez les deux espèces la provision de pollen est consommée en 2 à 5 semaines. Les 3 premiers stades larvaires sont très brefs : premier stade, 1 jour; deuxième stade, 1 à 2 jours; troisième stade, 4 à 5 jours; le quatrième stade dure environ 82 jours chez $O$. cornuta et 50 chez $O$. rufa.

\section{L'évolution de la morphologie et du comportement au cours du développement}

Chez les deux espèces, l'œuf, légèrement incurvé, mesure 4 à $5 \mathrm{~mm}$ de long et $1,5 \mathrm{~mm}$ de diamètre. L'extrémité céphalique est effilée. L'œuf nouvellement pondu est hyalin, mais au bout de 2 ou 3 jours on distingue dorsalement une zone laiteuse légèrement annelée qui est l'embryon. La segmentation devient de plus en plus nette au fur et à mesure que l'on se rapproche de l'éclosion (fig. 1 - A, B, C). La jeune larve se débarrasse du chorion et touche de la tête le pollen après une série de balancements de haut en bas de la partie antérieure du corps. La partie postérieure reste fixée dans la boule de pollen (fig. $1-D$ ). Peu à peu le pollen ingéré colore le tube digestif de la larve dont les téguments sont transparents. Le $2^{\mathrm{e}}$ et le $3^{\mathrm{e}}$ stade larvaire, de petite taille et de courte durée ne consomment pas une grande quantité de « pain d'abeille ). 


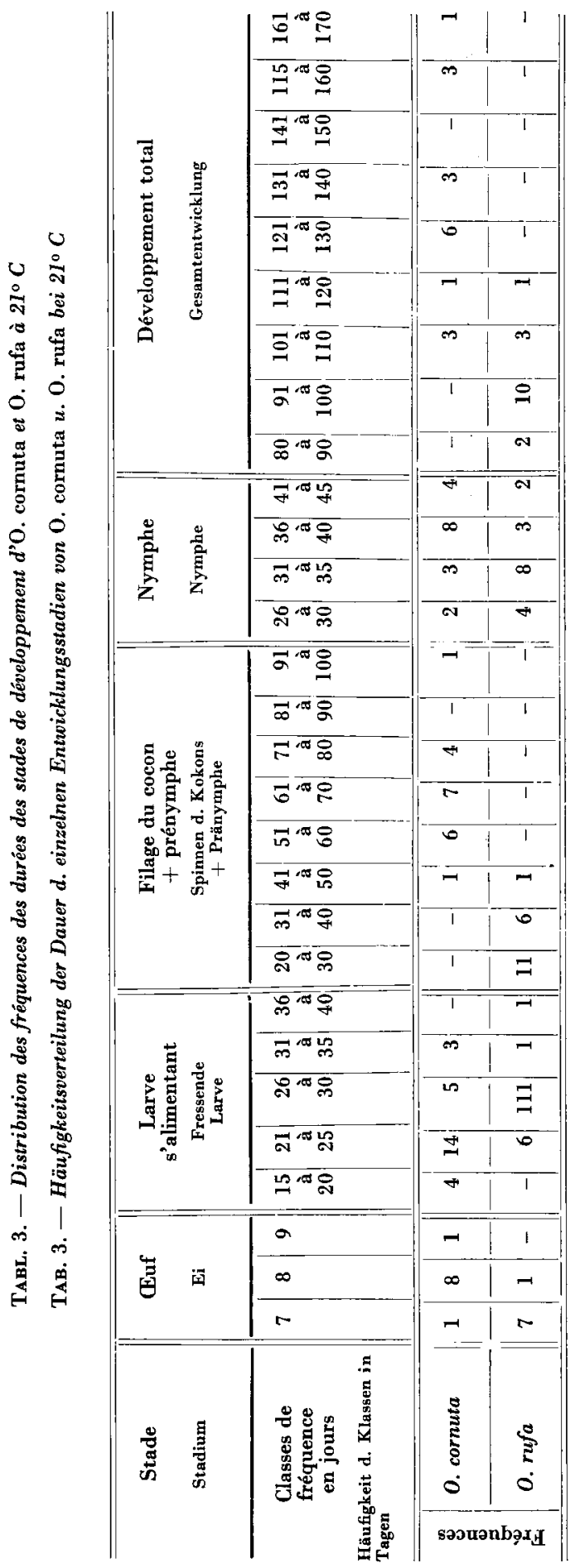



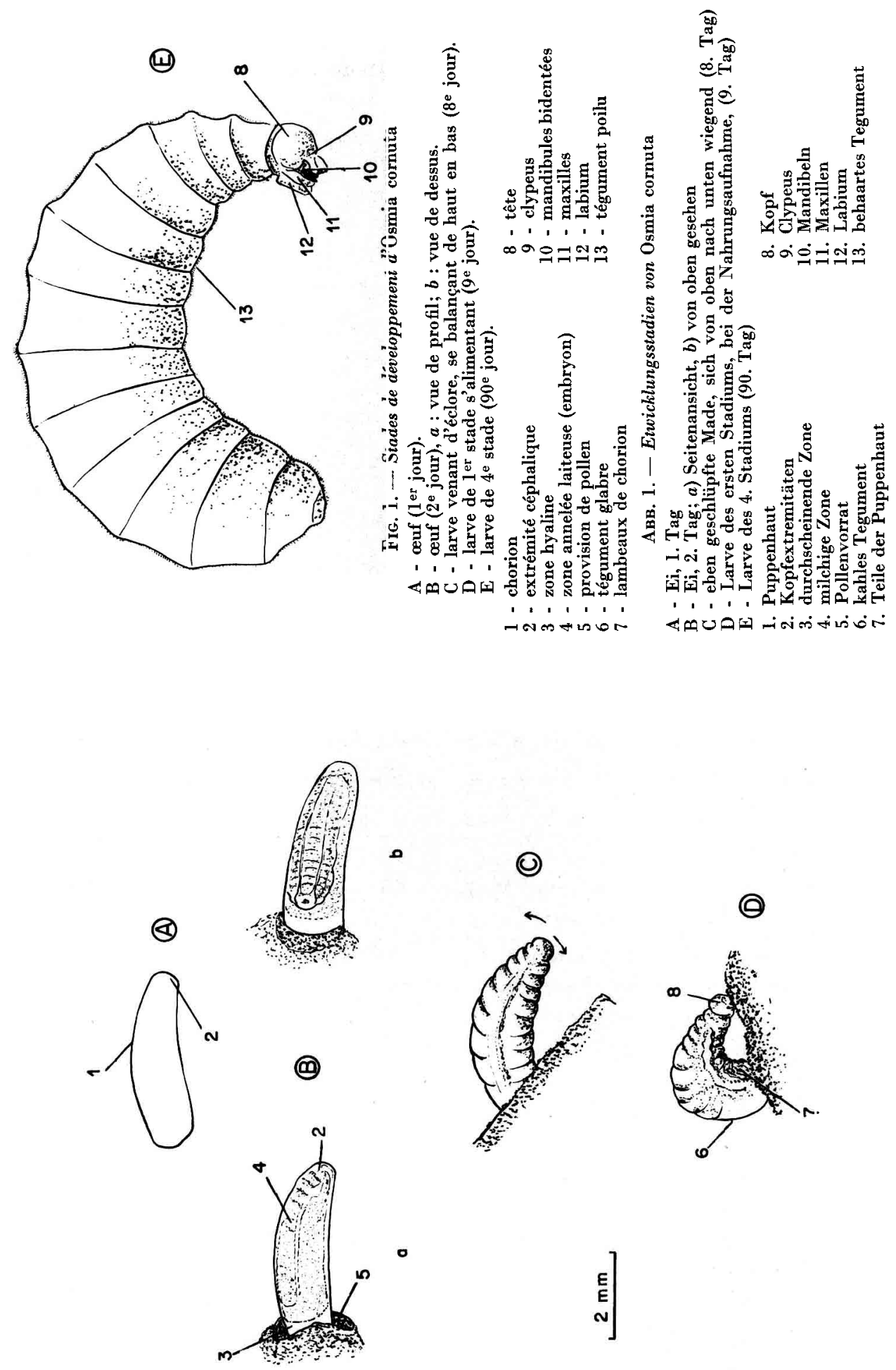
La $3^{\mathrm{e}}$ mue larvaire laisse apparaître le $4^{\mathrm{e}}$ stade (fig. $1-\mathrm{E}$ ), qui est poilu comme chez tous les Megachilidae. C'est à partir de ce moment c'est-à-dire 6 à 7 jours après l'éclosion, qu'a lieu l'expulsion des excréments. Il faut 15 à 20 jours pour que le $4^{\mathrm{e}}$ stade larvaire d'O. cornuta termine les provisions alors que 20 à 30 jours sont nécessaires au $4^{\text {e }}$ stade larvaire d' $O$. rufa pour la même opération. D'après Malyshev (1935) les larves peuvent aussi manger leurs exuvies qui s'accumulent, chiffonnées, à l'extrémité postérieure et ventrale de leur corps.

La larve âgée d' $O$. cornuta et d' $O$. rufa mesure 9 à $13 \mathrm{~mm}$ de long. Elle commence à filer son cocon quand les provisions sont épuisées. L'activité de filage dure de 7 à 20 jours. Chez les deux espèces le cocon est composé de plusieurs couches (Losinski, 1936).

Chez $O$. cornuta la couche externe est un feutrage brun très clair enveloppant le cocon entièrement :

- la $2^{\mathrm{e}}$ couche est fine mais résistante, de couleur brun rouge clair.

- la $3^{\mathrm{e}}$ couche est un feutrage brun clair assez dense.

- la $4^{\mathrm{e}}$ couche est épaisse, brun rouge foncé, et revêtue sur sa face interne d'un vernis très mince qui peut se détacher par plaques.

Chez $O$. rufa la couche externe est presque inexistante. Le feutrage blanc est extrêmement lâche tout autour du cocon. Il ne devient dense qu'à la base du mamelon situé à la partie antérieure du cocon.

- la $2^{\mathrm{e}}$ couche brun rouge clair est fine et résistante.

- la $3^{\mathrm{e}}$ couche brun rouge foncé est la plus épaisse et la plus résistante de toutes.

- la $4^{\mathrm{e}}$ couche brun rouge clair est fine et résistante. Elle est plus vernissée sur sa surface interne.

Un simple et rapide examen des cocons permet de différencier ceux qui sont filés par $O$. cornuta et ceux qui sont filés par $O$. rufa : l'ouvrage de la première espèce a une couleur plus claire que celui d'O. rufa, à cause de la présence d'un important feutrage externe. De plus il résiste moins bien aux pressions, les couches internes étant moins rigides que celles constituant l'ouvrage $d^{\prime} O$. rufa. Chez les deux espèces on reconnaît la partie antérieure du cocon à la présence d'un mamelon dont la base mesure 1 à $2 \mathrm{~mm}$ de diamètre et dont la hauteur ne dépasse pas $1 \mathrm{~mm}$. Bien que les cocons renfermant les femelles soient souvent plus gros que ceux renfermant les mâles, on ne trouve pas de différence significative entre les poids ou les longueurs des cocons mâles et des cocons femelles. (Lecomte - communication personnelle).

Après filage du cocon la larve se couche sur le dos, en arc de cercle et reste ainsi immobile jusqu'à la mue nymphale. La nymphe (fig. 2) est aussi un stade immobile. A partir de ce moment on peut distinguer les mâles des femelles, 

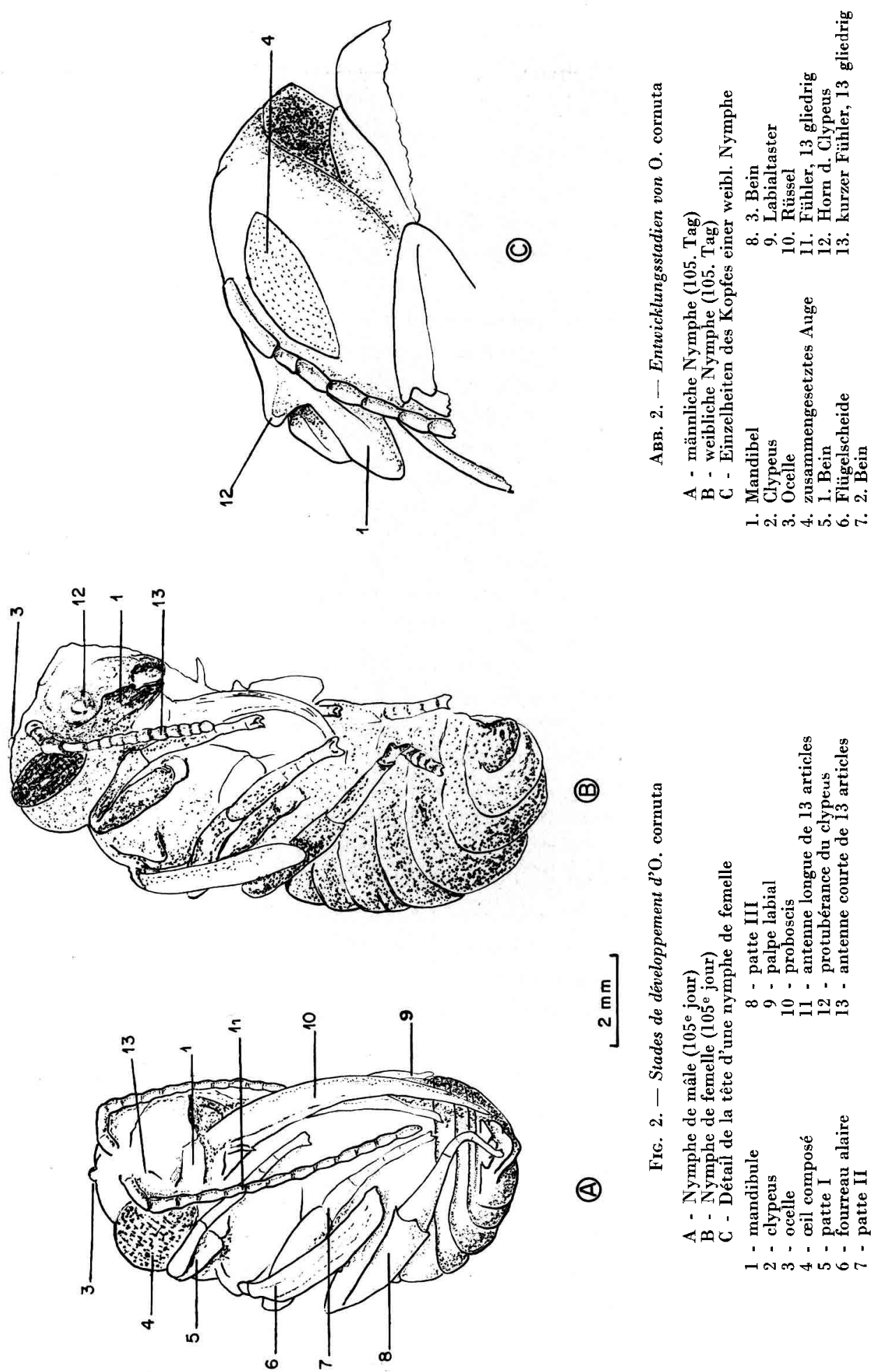
grâce aux protubérances du clypeus qui existent chez les femelles des deux espèces (fig. 2, C). On distingue d'ailleurs les femelles des deux espèces au stade imago, par l'examen de ces protubérances (fig. 3). Pendant un à dix jours la nymphe reste entièrement blanche, puis les yeux deviennent roses et leur couleur ne cesse de s'assombrir pendant deux ou trois semaines. Enfin les mandibules se pigmentent à leur tour puis les ailes et le corps tout entier, jusqu'au moment de la mue imaginale. La progression de la pigmentation de la nymphe dure 8 à 18 jours.

Le développement d'O. cornuta et d'O. rufa est assez semblable à celui d'O. lignaria (Levin, 1966 - Krombein, 1962) : Michener (1953) affirme qu'il y a peu de différence entre une larve d' $O$. rufa et une larve d'O. lignaria. Il est bon de rappeler que les 3 espèces $O$. cornuta, $O$. rufa, $O$. lignaria appartiennent au même sous-genre Osmia.

\section{Les causes de mortalité chez les stades immatures}

Le tableau 4 indique de façon globale la mortalité chez $O$. cornuta et $O$. rufa en conditions contrôlées (œufs obtenus en serre) et en conditions naturelles (œufs obtenus dans les nichoirs extérieurs).

La mortalité totale est très élevée chez les deux espèces, de l'ordre de $60 \%$ des œufs pondus. Le parasitisme cause 17 à $21 \%$ de perte, ce qui est peu si l'on considère les taux de perte due à des causes indéterminées (œufs, larves, nymphes se desséchant sans que l'on observe d'être vivant parasite ou prédateur dans la cellule). Ce sont surtout les larves qui sont atteintes par cette maladie. Les adultes meurent très rarement avant le début de l'hibernation. Des taux importants de mortalité due à des causes inconnues sont rapportés par divers auteurs étudiant d'autres espèces d'osmies : MaEta (1965), Maeta et Kitamura (1968), Danks (1971), Tasei (1972), Raw (1972).

Les êtres vivants causant des ravages dans les nids des deux espèces à Lusignan sont des moisissures, un Hyménoptère Eulophidae : Melittobia acasta Walk. et un Diptère, Cacoxenus indagator Loew (Drosophilidae).

Melittobia acasta parasite beaucoup d'autres Osmies, Megachilides et Apoïdes. Elle s'attaque même aux guêpes solitaires et à des tachinaires parasites de ces guêpes, (Grasse, 1951). Hardouin (1948) a observé $M$. acasta sur Osmia cornuta, O. rufa, O. coerulescens. Maréchal (1926) sur Osmia aurulenta. Plusieurs auteurs : Picard (1922), Parker et Thompson (1928) et surtout Balfour-Browne (1922) ont étudié la biologie de ce petit ectoparasite. Les adultes mesurant un peu plus de $1 \mathrm{~mm}$ de long se rencontrent de mai à septembre. Les mâles sont aveugles et peu nombreux ( 1 à $5 \%$ de la population totale). Les femelles piquent leur hôte avec leur tarière; il y a ainsi formation d'un (c tube nutritif ) qui permet à Melittobia d'aspirer le sang de l'insecte mort ou paralysé. La ponte a lieu sur des prénymphes ou des nymphes. Une 

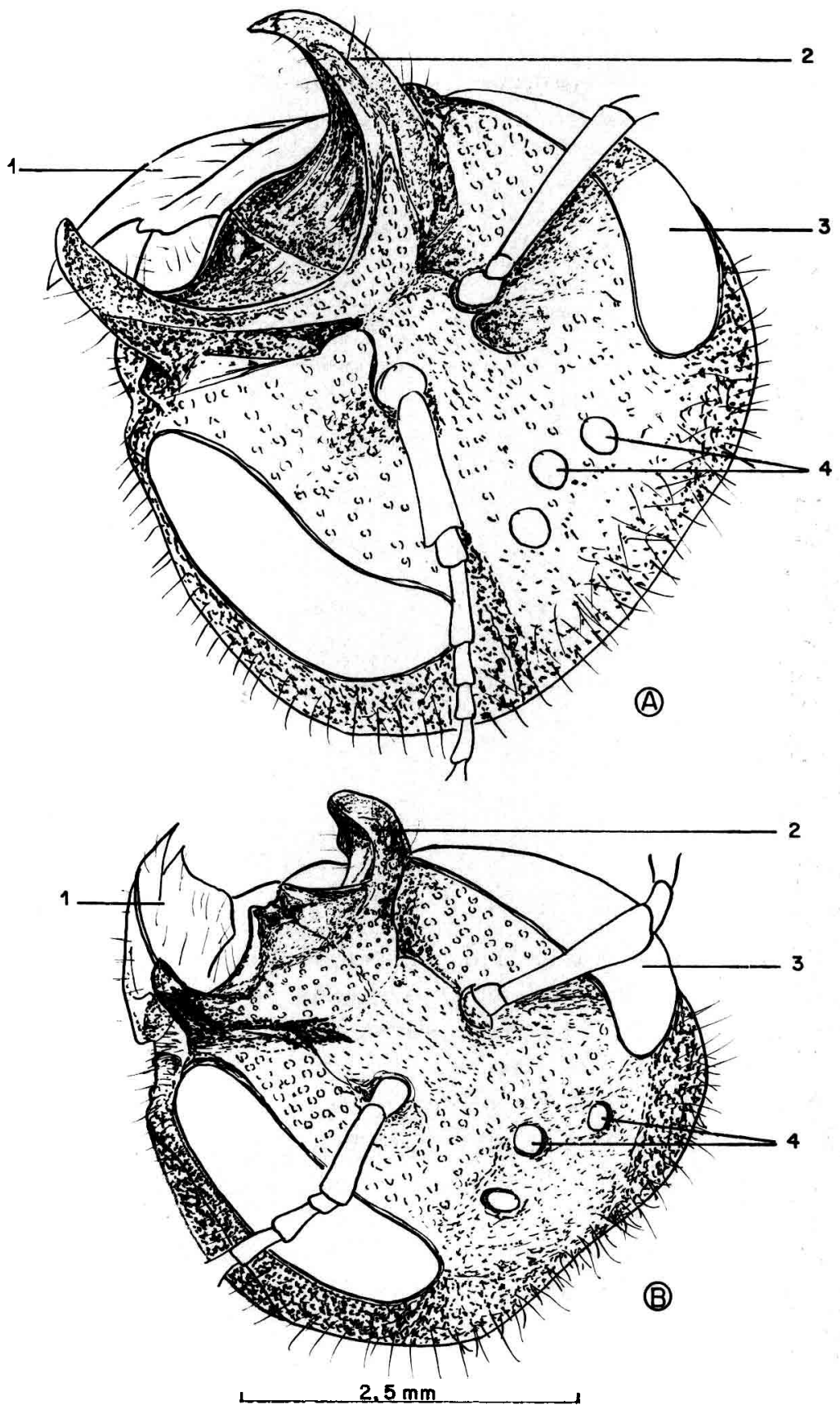

Fic. 3. - Têtes d'imagos femelle vues de face

Aвв. 3. - Köpfe weibl. Imagines, Vorderansicht

$$
\begin{aligned}
& \text { A - O. cornuta } \\
& \text { B - O. rufa }
\end{aligned}
$$

1 - mandibule

2 - corne du clypeus

3 - ceil composé

4. - ocelles

$$
\begin{aligned}
& \text { A - O. cornuta } \\
& \text { В - O. rufa }
\end{aligned}
$$

1. Mandibel

2. Clypeus-Horn

3. zusammengesetztes Auge

4. Ocellen 
TaBl. 4. - Mortalité chez O. rufa et O. cornuta (1970-1971). (Stades immatures)

TAB. 4. - Sterblichkeit bei O. rufa $u$. O. cornuta (1970-1971). (Larvenstadien)

\begin{tabular}{|c|c|c|c|c|c|}
\hline & & $\begin{array}{c}\text { Nombre } \\
\text { d'œufs } \\
\text { pondus } \\
\text { Zahl der } \\
\text { gelegten Eier }\end{array}$ & $\begin{array}{c}\text { Mortalité due } \\
\text { à des causes } \\
\text { indéfinies } \\
\text { Sterblichkeit aus } \\
\text { unbekannter } \\
\text { Ursache }\end{array}$ & \begin{tabular}{|c|} 
Mortalité due \\
à des parasites \\
et des moisissures \\
Sterblichkeit, \\
verursacht durch \\
Parasiten u. \\
Schimmel
\end{tabular} & $\begin{array}{l}\text { Mortalité } \\
\text { totale } \\
\text { Gesamtzahl } \\
\text { d. Todesfälle }\end{array}$ \\
\hline $\begin{array}{c}\text { oufs } \\
\text { obtenus } \\
\text { dans la } \\
\text { nature }\end{array}$ & $\begin{array}{c}\text { O. rufa } \\
1970 \\
1971 \\
\text { Total } \\
\%\end{array}$ & $\begin{array}{r}66 \\
97 \\
163 \\
-\end{array}$ & $\begin{array}{c}33 \\
38 \\
71 \\
(43,5)\end{array}$ & $\begin{array}{c}8 \\
20 \\
28 \\
(17,2)\end{array}$ & $\begin{array}{c}41 \\
58 \\
99 \\
(60,7)\end{array}$ \\
\hline Bedingungen & $\begin{array}{c}\text { O. cornuta } \\
1971 \\
\%\end{array}$ & $\begin{array}{l}42 \\
-\end{array}$ & $\begin{array}{c}17 \\
(40,5)\end{array}$ & $\begin{array}{c}9 \\
(21,5)\end{array}$ & $\begin{array}{c}26 \\
(62,0)\end{array}$ \\
\hline $\begin{array}{c}\text { ceufs } \\
\text { obtenus } \\
\text { en serre }\end{array}$ & $\begin{array}{c}\text { O. rufa } \\
1970\end{array}$ & 43 & 17 & - & 17 \\
\hline $\begin{array}{l}\text { Zahl d. Eier } \\
\text { im Treibhaus }\end{array}$ & $\begin{array}{l}\text { O. cornuta } \\
1970\end{array}$ & 13 & 6 & - & 6 \\
\hline
\end{tabular}

femelle peut pondre plus d'un millier d'œufs tout au long de ses 3 ou 4 mois d'activité. Elle passe d'une cellule d'Osmie à une autre. Il peut y avoir jusqu'à 6 générations par an. L'hibernation s'effectue au stade larvaire, rarement au stade nymphal. Après l'accouplement les femelles ont tendance à sortir de la cellule où elles ont effectué leur développement, puis un puissant « chemiotropisme " les conduit vers le nid de leur hôte où elles se laissent murer.

Cacoxenus indagator est un cleptoparasite assez fréquent dans les nids d'Osmies : $O$. rufa et $O$. cornuta (MARÉCHAL, 1932); O. rufa, O. cornuta et O. coerulescens (Hardouin, 1948); O. rufa, O. cornuta, O. coerulescens, O. ventralis (Julliard, 1947 et 1948); O. rufa (Raw, 1972). Julliand a étudié la biologie et le comportement de l'insecte : les cellules parasitées contiennent plusieurs larves, serrées les unes contre les autres, qui dévorent le pollen et remplissent leur espace vital d'excréments filamenteux très longs, de couleur brun rouge. Il peut arriver que les asticots mangent la jeune larve d'Osmie. Par contre s'ils sont peu nombreux la larve d'Osmie survit mais reste très petite. Les larves de Cacoxenus passent parfois d'une cellule à l'autre en perçant les cloisons avec leurs crochets buccaux. Lorsque les provisions sont consommées, les larves entrent en diapause. Au printemps ces larves se trans- 
forment en pupes 2 à 3 semaines avant l'émergence, qui a toujours lieu après le départ des Osmies.

\section{B. - L'influence des températures hivernales sur les délais d'émergence des adultes}

A la fin de l'été les adultes d'O. cornuta et d'O. rufa sont apparemment prêts à s'envoler. Si l'on fait des dissections d'appareils reproducteurs mâle à diverses périodes on s'aperçoit que la spermatogenèse a lieu avant la mue imaginale et qu'à l'automne le sperme s'accumule progressivement dans les vésicules séminales des adultes. Aux mois de novembre et décembre les tubes spermatiques d'O. cornuta sont absolument vides et ceux d'O. rufa sont en train de se vider. Si à ce moment on porte les cocons à une température constante de l'ordre de $20^{\circ} \mathrm{C}$ la plupart d'entre eux meurt et les rares individus qui émergent après plusieurs semaines ne sont pas aptes à voler normalement (Lecomte, 1966). Au contraire si on les laisse dans un endroit froid pendant 2 ou 3 mois il est possible d'obtenir, dans des délais beaucoup plus courts, la sortie de la grande majorité des insectes qui ont dans ce cas une activité normale. Ceci prouve que si le développement des organes externes et internes de ces osmies semble achevé, la maturation physiologique des adultes demande encore plusieurs mois d'hiver que les insectes passent immobiles dans leur cocon. On peut dire que le développement imaginal d'O. cornuta et $O$. rufa est obligatoirement arrêté, tant que les insectes n'ont pas subi une périope de froid. C'est un caractère qui se retrouve dans tous les cas de diapause.

Le but de mon travail n'est $\boldsymbol{\rho}$ s de mettre en évidence les facteurs qui induisent cet engourdissement physiologique, ni les processus métaboliques accompagnant l'élimination de la diapause. J'ai simplement voulu montrer quelle est l'influence respective de quelques traitements thermiques sur les délais d'émergence des deux espèces.

\section{Résultats}

Les traitements des différents lots d'osmies ayant servi aux expériences d'hibernation sont indiqués sur le tableau 5. Les effets de ces traitements sur les délais d'émergence sont représentés sur les figures 4 et 5 et sur les tableaux 6 et 7 .

Le lot témoin composé de 20 osmies n'hibernant pas, s'est comporté de la façon suivante :

- 5 mâles d'O. cornuta ont émergé après 45-48-50-57 et 60 jours respectivement. 

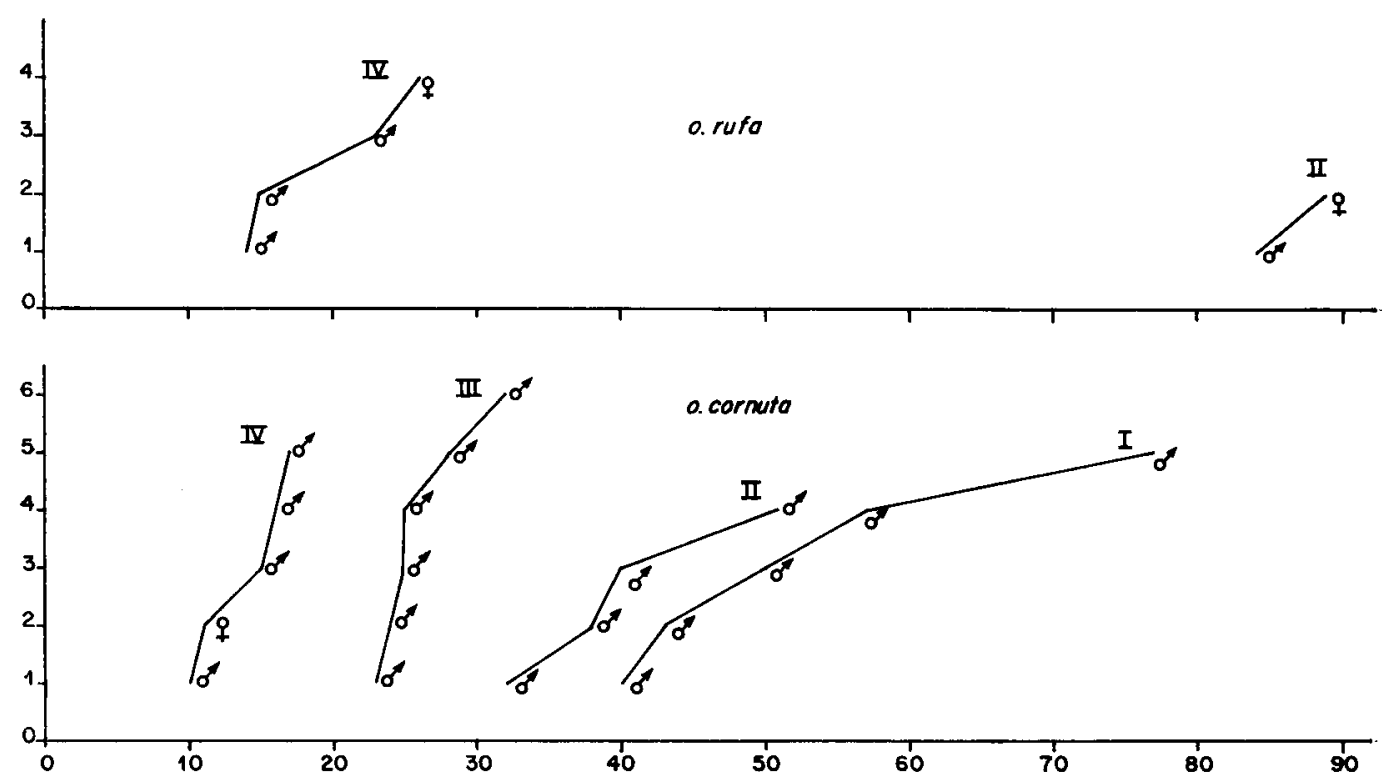

Frg. 4. - Emergences des osmies ayant hibernè à $0^{\circ} \mathrm{C}$ axe des $\mathrm{x}=$ durée de réactivation à $20^{\circ} \mathrm{C}$ en jours axe des $y=$ ordre d'émergence

chiffres romains = lots expérimentaux (cf. : tab. 5)

${ }^{x}$, ou $q=$ un individu mâle ou femelle.

Авв. 4. - Das Schlüpfen von Osmien, die bei $0{ }^{\circ} \mathrm{C}$ überwintert haben

$\mathrm{X}$-Achse $=$ Dauer der Reaktivierung bei $20^{\circ} \mathrm{C}$ (in Tagen)

Y-Achse $=$ Reihenfolge des Schlïpfens

Römische Ziffern = Versuchsgruppen (S. Tab. 5)

$\star$ oder ㅇ $=$ männliche oder weibliche Individuen morts.

- 3 mâles d'O. rufa après 60-69 et 84 jours. Les 12 autres individus sont

Ces résultats, bien qu'étant obtenus avec des lots expérimentaux numériquement faibles, me permettent de tirer quelques conclusions et de les discuter.

\section{Conclusions}

a) Lorsque les deux espèces sont soumises aux mêmes conditions de température, en particulier aux mêmes temps d'hibernation à $0^{\circ}$ ou à $4^{\circ} \mathrm{C}, O$. cornuta émerge en moyenne 15 à 20 jours plus tôt qu'O. rufa (Fig. 5). Cela n'est pas surprenant puisque ce décalage des sorties se produit aussi dans les conditions naturelles (TASEI, sous presse).

b) La température de $4^{\circ} \mathrm{C}$ semble plus favorable à la survie des insectes que la température de $0^{\circ} \mathrm{C}$ (cf : tableau 5). 
c) L'allongement de la période d'hibernation entraine une diminution du délai moyen de réactivation. $D$ 'autre part il y a une très forte corrélation
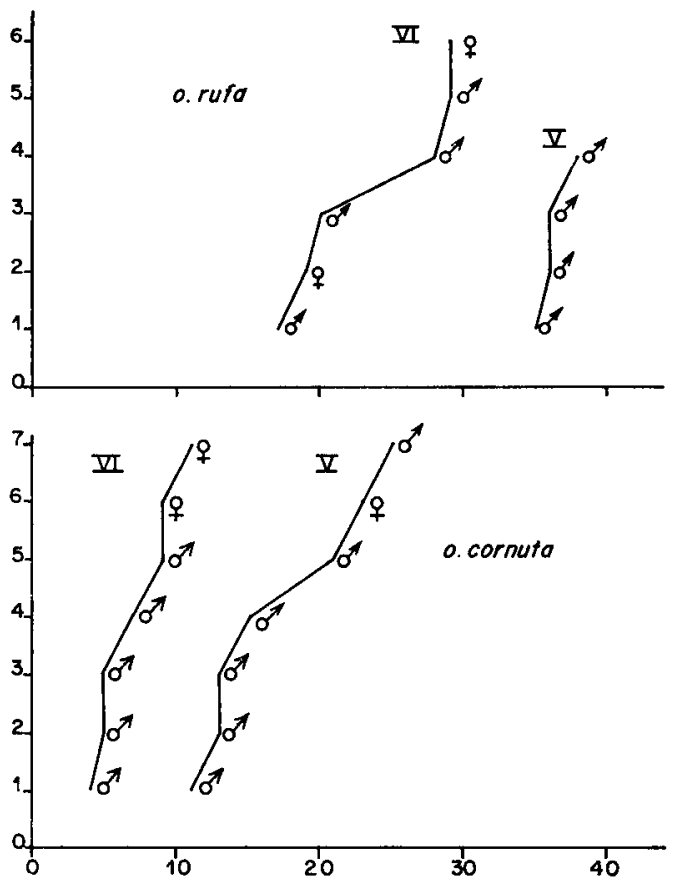

Fig. 5. - Emergences des Osmies ayant hiberné à $4{ }^{\circ} \mathrm{C}$ même légende que pour la Fig. 4.

Aвв. 5. - Schlupf der Osmien, die bei $4^{\circ} \mathrm{C}$ überwintert haben sonst wie bei Abb. 4

TABL. 5. - Lots d'insectes servant à étudier les réactions des Osmies aux traitements thermiques

TaB. 5. - Gruppen von Osmien, die für die Untersuchung ihrer Reaktionen auf Temperatureinwirkungen bestimmt sind

\begin{tabular}{|c|c|c|c|c|c|c|}
\hline \multirow[t]{2}{*}{$\begin{array}{c}\text { Température } \\
\text { d'hibernation } \\
\text { Uberwinterungs- } \\
\text { temperatur }\end{array}$} & \multirow[t]{2}{*}{$\begin{array}{c}\text { Durée } \\
\text { d'hibernation } \\
\text { en jours } \\
\text { Dauer d. Uber- } \\
\text { winterung in Tagen }\end{array}$} & \multirow[t]{2}{*}{$\begin{array}{l}\text { No lot } \\
\text { Nr. der } \\
\text { Gruppe }\end{array}$} & \multicolumn{2}{|c|}{$\begin{array}{l}\text { Nombre de cocons } \\
\text { le } 1 \text { er décembre } \\
\text { Anzahl d. Kokons } \\
\text { am } 1 . \text { Dez. }\end{array}$} & \multicolumn{2}{|c|}{$\begin{array}{c}\text { Nombre d'émergences } \\
\text { à la température } \\
\text { de } 20^{\circ} \\
\text { Zahl d. geschlüpften } \\
\text { Tiere b. } 20^{\circ}\end{array}$} \\
\hline & & & O. cornuta & O. rufa & O. cornuta & o. $r u f a$ \\
\hline $0^{\circ}$ & $\begin{array}{l}15 \\
33 \\
61 \\
93\end{array}$ & $\begin{array}{r}\text { I } \\
\text { II } \\
\text { III } \\
\text { IV }\end{array}$ & $\begin{array}{l}7 \\
5 \\
9 \\
5\end{array}$ & $\begin{array}{l}\overline{8} \\
\overline{6} \\
\end{array}$ & $\begin{array}{l}5 \\
4 \\
6 \\
5\end{array}$ & $\begin{array}{l}-\overline{2} \\
\overline{4}\end{array}$ \\
\hline $4^{0}$ & $\begin{array}{r}73 \\
110\end{array}$ & $\begin{array}{r}\text { V } \\
\text { VI }\end{array}$ & $\begin{array}{l}7 \\
7\end{array}$ & $\begin{array}{l}4 \\
6\end{array}$ & $\begin{array}{l}7 \\
7\end{array}$ & $\begin{array}{l}4 \\
6\end{array}$ \\
\hline
\end{tabular}


TABL. 6. - Caractéristiques des émergences des lots expérimentaux d'O. cornuta

TAB. 6. - Besonderheiten beim Schlüpfen der Versuchsgruppen von 0. cornuta

\begin{tabular}{|c|c|c|c|c|}
\hline \multicolumn{2}{|l|}{$\mathbf{A}$} & $\mathbf{B}$ & $\mathbf{A}+\mathbf{B}$ & \\
\hline \multicolumn{2}{|c|}{$\begin{array}{l}\text { Durées de l'hibernation } \\
\text { artificielle (en jours) }\end{array}$} & $\begin{array}{l}\text { Durées moyennes de la } \\
\text { réactivation à } 20^{\circ} \\
\text { (en jours) }\end{array}$ & $\begin{array}{c}\text { Délais d'émergence } \\
\text { (à partir } \\
\text { du ler décembre) }\end{array}$ & $\begin{array}{c}\text { Dispersion des sorties } \\
\text { dans le temps } \\
\text { (en jours) }\end{array}$ \\
\hline \multicolumn{2}{|c|}{$\begin{array}{l}\text { Dauer d. künstl. } \\
\text { Überwinterung } \\
\text { (in Tagen) }\end{array}$} & $\begin{array}{l}\text { Durchschnittl. } \\
\text { Dauer d. Reaktivierung } \\
\text { (in Tagen) }\end{array}$ & $\begin{array}{c}\text { Verzögerung d. } \\
\text { Schlüpfens } \\
\text { (vom 1. 12. ab) }\end{array}$ & $\begin{array}{l}\text { Zeitl. Verteilung } \\
\text { d. Schlïpfens } \\
\text { (in Tagen) }\end{array}$ \\
\hline Lot témoin & 0 & & 50 & 15 \\
\hline à $0^{0}$ & $\begin{array}{l}15 \\
33 \\
61 \\
93\end{array}$ & $\begin{array}{l}53 \\
40,5 \\
26 \\
14\end{array}$ & $\begin{array}{l}68 \\
73,5 \\
87 \\
107\end{array}$ & $\begin{array}{r}38 \\
19 \\
9 \\
8\end{array}$ \\
\hline à $4^{0}$ & $\begin{array}{r}73 \\
110\end{array}$ & $\begin{array}{c}17 \\
7,1\end{array}$ & $\begin{array}{c}90 \\
117,1\end{array}$ & $\begin{array}{r}14 \\
7\end{array}$ \\
\hline
\end{tabular}

TABL. 7 - Emergences d'Osmia cornuta (mâles) selon l'origine géographique.

$M=$ individu originaire du Sud de la France

$\mathrm{N}=$ individu originaire du Nord de la France

$\|=$ Marque le milieu de la période des émergences de chaque lot

TAB. 7. - Schlüpfen von O. cornuta (Männchen) ihrem geographischen Ursprung entsprechend

$M=$ Tiere aus Südfrankreich

$\mathbf{N}=$ Tiere aus Nordfrankreich

$\|=$ Milieu während der Sehlüpfperiode jeder Gruppe

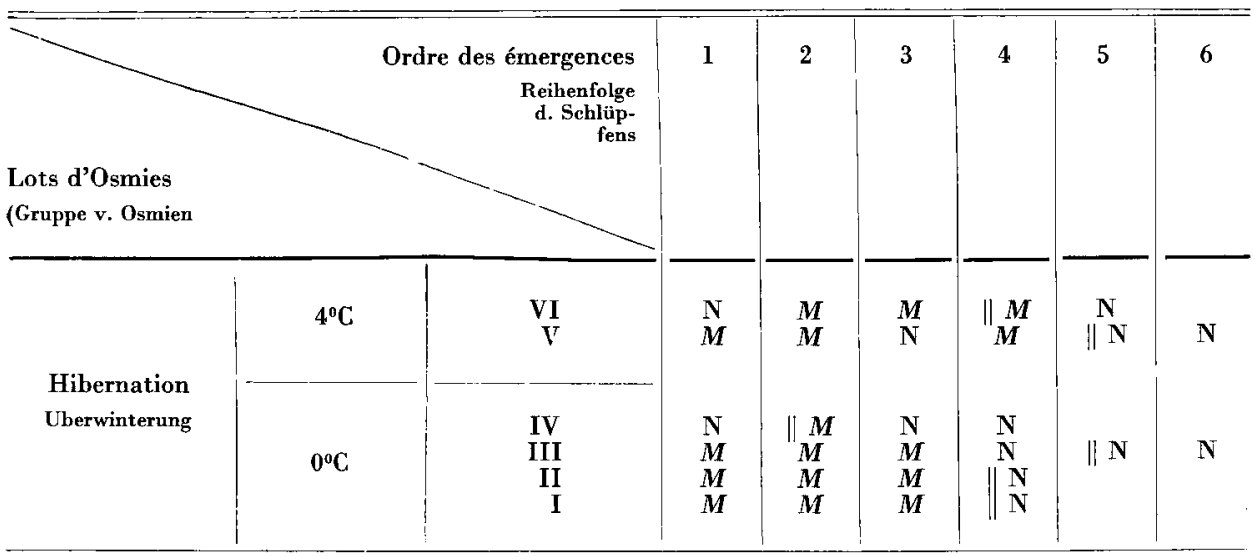


négative entre la longueur de l'hibernation et la dispersion des émergences dans le temps. Il est bon de remarquer que l'allongement de la période d'hibernation entraîne un allongement de la période comprenant l'hibernation et la réactivation. Les rapports existant entre les durées d'hibernation et les durées de réactivation sont aussi relatés chez Thersilochus melanogaster Thoms. (Hymenoptera Ichneumonidae) par JourdheuIL (1961), et chez Megachile rotundata F. (Hymenoptera Megachilidae) par JoHAnsen et Eves (1973).

d) La comparaison des mortalités en élevage de 1966 et 1967 (conditions semi-contrôlées pendant la diapause) et des mortalités en élevage de 1970 (conditions entièrement contrôlées) met en évidence la supériorité de l'élevage en conditions semi-contrôlées et l'importance de la période de préhibernation, qui succède à la mue imaginale et précède l'hibernation proprement dite. Une préhibernation à température élevée $\left(20^{\circ} \mathrm{C}\right)$ ne permet pas aux Osmies de supporter une température basse. Une préhibernation ayant lieu dans les conditions naturelles jusqu'aux mois d'octobre et novembre permet aux Osmies de subir l'hibernation à basse température. Ces observations sont à rapprocher de celles de Pouvreau (1970). Cet auteur remarque que les reines de bourdons (Bombus Latr.) ont aussi besoin d'une période “ d'acclimatation » aux basses températures; il acclimate les reines en les soumettant à des températures décroissantes de $17^{\circ}$ à $4^{\circ} \mathrm{C}$, reproduisant ainsi les conditions naturelles.

Krunic et Hinks (1972) affirment également que Megachile rotundata F. (Hymenoptera Megachilidae) supporte mieux les basses températures d'hibernation si les prénymphes en diapause sont progressivement acclimatées par des séjours d'une semaine aux températures : $15^{\circ}, 10^{\circ}, 5^{\circ}, 0^{0},-5^{\circ} \mathrm{C}$.

e) Le tableau 7 distingue pour chaque lot expérimental 2 périodes : la première est comprise entre l'émergence la plus précoce et le milieu de la période des émergences du lot; la deuxième est comprise entre le milieu de cette période et la dernière émergence.

14. Osmies méridionales et seulement 4 Osmies nordiques ont émergé pendant la première période de tous les lots, tandis que 9 Osmies nordiques et seulement 3 Osmies méridionales ont émergé pendant la deuxième période. Cela signifie que les individus méridionaux sont réactivés en moyenne plus rapidement que les nordiques, bien qu'ayant hiberné de façon identique. Or ces insectes ayant des origines géographiques différentes n'ont pas de mues imaginales synchrones. Les individus méridionaux ont une phase de préhibernation plus longue que celle des individus septentrionaux. On peut supposer que les premiers ont atteint un degré de c développement de diapause » supérieur, lorsque commencent les expériences de laboratoire. Ces individus bénéficieraient donc davantage des traitements thermiques d'hibernation et de réactions contrôlées. 
Une deuxième hypothèse peut être formulée : il existerait 2 races géographiques d' $O$. cornuta et leur diapause se déroulerait d'une manière légèrement différente dans chaque cas.

\section{CONCLUSIONS}

1) On a mis en évidence des caractères physiologiques et morphologiques différenciant $O$. cornuta d'O. rufa :

- Le développement de la première espèce demande environ 5 semaines de plus que celui d'O. rufa.

- Le $4^{\mathrm{e}}$ stade larvaire est presque 2 fois plus long chez $O$. cornuta que chez 0 . rufa.

- Les cocons d'O. cornuta sont revêtus d'un feutrage blanc, que ne possèdent pas les cocons d'O. rufa.

2) Les deux espèces ont quelques caractères communs :

- La mue imaginale se produit en fin d'été ou à l'automne.

- La mortalité est très élevée chez les stades immatures.

- Le parasitisme est dû à Lusignan à Melittobia et Cacoxenus.

3) Les deux espèces d'Osmies sont monovoltines. Leur diapause imaginale est éliminée par le froid. Si l'hibernation est longue les émergences sont plus précoces et mieux synchronisées que si l'hibernation est courte. $O$. rufa réagit moins rapidement qu'O. cornuta; elle est réactivée plus lentement que cette dernière espèce.

La phase de préhibernation doit obligatoirement être une période d'acclimatation à la température d'hibernation. Pendant cette phase la température doit être comprise entre la température de développement et celle d'hibernation.

De nouvelles investigations seraient nécessaires si l'on voulait définir le meilleur mode de préhibernation, la gamme des températures d'hibernation et l'influence de l'origine géographique des individus sur la rapidité de l'élimination de la diapause. Enfin une étude spéciale pourrait mettre en évidence le facteur inducteur de cet arrêt de développement.

Reçu pour publication en juin 1973.

Eingegangen im Juni 1973.

\section{REMERCIEMENTS}

Je tiens à remercier M. R. de SACY (I.N.R.A. Versailles) qui a identifié le parasite Cacoxenus indagator Loew. 


\section{ZUSAMMENFASSUNG}

In einer früheren Arbeit (TASEI, im Druck) habe ich die Ergebnisse einer vergleichenden Studie über das Verhalten von $O$. cornuta und $O$. rufa beim Nestbau dargestellt. Die vorliegende Arbeit berichtet über die Entwicklung der Larvenstadien und über die Diapause beider Arten.

\section{A. - Entwicklung der Larvenstadien}

Die Nester der Osmien verdanken wir der Anlage von Fallennestern, die mit Rohrstengeln ausgestattet wurden (S. TASEI, 1972). Die Nester wurden in zwei Gruppen aufgeteilt : die erste Gruppe blieb im Freien in einem hölzernen Schutzhäuschen; die zweite wurde konstant bei $21^{\circ} \mathrm{C}$ gehalten. Die Kontrolle der Entwicklung wurde in der ersten Gruppe alle zwei Wochen, in der zweiten jeden Tag durchgeführt. In den Tabellen 1, 2, 3, 4 sind die Beobachtungsergebnisse dargestellt.

1. - Osmia cornuta entwickelt sich langsamer als Osmia rufa. Unter den Bedingungen von Lusignan fällt die Periode der Imaginalhäutung im Freien in die Zeit zwischen dem 15. August und Ende Oktober (Tab. 1).

2. - Bei einer Temperatur von $21^{\circ} \mathrm{C}$ dauert bei $O$. cornuta die Entwicklung vom Ei bis zur Imago 19 Wochen, bei $O$. rufa 14 Wochen. Bei beiden Arten wird der Pollenvorrat in 2 bis 5 Wochen aufgezehrt. Das 4. Larvenstadium dauert bei 0 . cornuta ungefähr 82 Tage, bei o. rufa 50 Tage (Tab. 2).

3. - Die morphologische Entwicklung der Larvenstadien, vergleichbar für beide Arten, ist in Abb. 1 und 2 dargestellt. Die Kokons von $O$. rufa lassen sich durch ihren dichten, weissen Filzbelag leicht von den Kokons von $O$. cornuta unterscheiden.

4. - Während der ganzen Entwicklungszeit ist bei beiden Arten eine hohe Sterblichkeit zu verzeichnen. Schimmelpilze, eine Hymenoptere (Eulophidae) Melittobia acasta und eine Diptere (Drosophilidae) Cacoxenus indagator Loew verursachen 17 bis $21 \%$ der Verluste; während andere, nicht bestimmte Faktoren das Austrocknen von ungefähr $40 \%$ der Eier, Larven und Puppen verursachen.

\section{B. - Einfluss der Wintertemperaturen auf die Verzögerung des Schlüpfens der Adulten}

Osmia cornuta und Osmia rufa sind eimjährige Arten. Am Ende des Sommers können die offensichtlich zum Aus fliegen bereiten Imagines ihre Zellen nicht verlassen, wenn sie im Winter nicht tiefen Temperaturen ausgesetzt waren. Um den Einfluss des Winterschlafes auf das Schlüpfen zu untersuchen, wurden Kokons beider Arten aus Nistplätzen in verschiedenen Departements und aus Nestern von Chalicodomes (Megachile pyrenaica Lep.) im Südosten Frankreichs entnommen.

Wenn kein Winterschlaf stattfand, sterben die meisten Adulten ab; diejenigen, die schlüpfen, sind nicht imstande auszu fliegen. Lässt man die Kokons bei $4^{\circ} \mathrm{C}$ oder bei $0^{\circ} \mathrm{C}$ überwintern und reaktiviert bei $20^{\circ} \mathrm{C}$, erhält man folgende Ergebnisse :

1. - Nach Behandlung mit gleichen Temperaturen schlüpft $O$. cornuta früher als $O$. rufa (Abb. 5).

2. - Die Überwinterungstemperatur von $4{ }^{\circ} \mathrm{C}$ scheint günstiger als $0^{\circ} \mathrm{C}$ zu sein. 
3. - Die Verlängerung der Uberwinterungszeit verkürzt die durchschnittliche Verzögerung der Reaktivierung und bringt eine bessere Synchronisation des Schlüpfens mit sich (Tab. 6 und Abb. 4 und 5).

4. - Der Periode des eigentlichen Winterschlafes muss eine Phase des “ Vorschlafes 》 bei Temperaturen, die zwischen denen der Entwicklung und des Winterschlafes liegen, vorangehen.

5. - Die Individuen von $O$. cornuta südlichen Ursprungs scheinen früher zu schlüpfen als die nordischen Typen (Tab. 7). Sie hätten demnach eine grössere physiologische Reife, wenigstens dann, wenn es sich nicht um zwei geographische Rassen mit leicht abweichender Diapause handelt.

\section{RÉFÉRENCES BIBLIOGRAPHIQUES}

Balfour Bhowne F., 1922. - On the life history of Melittobia acasta Walker a chalcid parasite of bees and wasps. Parasitology, 14, 349-370.

Danks H. V., 1971. - Nest mortality factors in stem-nesting aculeate hymenoptera. J.animal Ecol., 40, 79-82.

Grasse P. P., 1951. - Traité de zoologie. Anatomie, systématique, biologie. Tome X, fasc. 1 : Névroptéroïdes, Hyménoptéroïdes, Mécoptéroïdes. Masson et Cie, Paris.

Hackwell G., 1968. - The biology and behaviour of the Alkalibee Nomia melanderi Cockerell. Oregon Stade University Ph. D.

Handouin R., 1948. - La vie des abeilles solitaires. 4'éd., Gallimard. Paris.

Johansen C. A., Eves J. D. 1973. - Effects of chilling, humidity and seasonal conditions on emergence of the alfalfa leafcutting bee. Environ. Entomol. 2, 23-26.

Joukdheuil P., 1961. - Influence de quelques facteurs écologiques sur les fluctuations de population d'une biocénose parasitaire. Thèse Fac. Sci. Univ., Paris.

Julliako C., 1947. - Contribution à l'étude d'un parasite (Cacoxenus indagator Loew., Drosophilidae) d'Osmia rufa L. Mitt. schweiz. entomol. Gesellsch. Bern., 20, 547-554.

JulliarD C., 1948. - - Le comportement des larves de Cacoxenus indagator dans les nids d'Osmia rufa. Mitt. schweiz. entomol. Gesellsch. Bern, 21, 547-554.

Krombein K. V., 1962. - Natural history of Plummers Island-Maryland-XVI-Biological notes on Chaetodactylus Krombeini Bak. a parasitic mite of Megachilid bee, Osmia (Osmia) lignaria Say. Proc. biol. Soc. Washington, 75, 237-249.

KRUNic M. D. et HiNks C. F., 1972. - The effect of temperature and of temperature pretreatment on diapause and on the synchronization of adult emergence in Megachile rotundata (Hym. Megachilidae) Canad. Entomol. 104, 889-893.

Leсомте J., 1966. - Communications personnelles.

LEVIN M. D., 1966. - Biological notes on Osmia lignaria and Osmia californica J. Kansas entomol. Soc., 39, 524-535.

Losınski P., 1936. — Ủber einen eigentümlichen Nestbau von Osmia bicornis L. Z. wiss. Insektenbiol., 7, 223-230 et 316-322.

MaETA Y., 1965. - Studies on the apple pollination by Osmia. Characteristics and underlaying problems in utilizing Osmia. Kontyu, 33, 17-34.

Maeta Y. et Kitamura T., 1968. - Some biological notes on the introduced wild bee, Osmia (Osmia) lignaria Say. (Hymenoptera, Megachilidae). Bull. Tohoku nat. agric. exper. Stn., 36, 53-70.

Malyshev S. I., 1935. - The nesting habits of solitary bees, A comparative study. Eos. XI. Lams II-XV.

Maréchal P., 1926. - Étude biologique d'Osmia aurulenta Panz. Bull. biol Fr. Belg., 60, 561-592. 
Maréchal P., 1932. - Recherches sur deux osmies communes : O. cornuta Latr. et $O$. rufa L. (Hym. Apid.). Bull Soc. entomol. Fr. 506-512.

Michener C. D., 1953. - Comparative morphological and systematic studies of bee larvae with a key to the families of Hymenopterous larvae. Univ. Kansas sci. Bull., 35, 987-1102.

Parker R. L. et Thompson W. R., 1928. - Biologie des Chalcidiens entomophages (Melittobia acasta Walk.). Ann. Soc. entomol. Fr., 97, 449-463.

PiCaro F., 1922. - Notes sur la biologie de Melittobia acasta Walk. Bull. Soc. entomol. Fr., 301-304.

Pouvreau A., 1970. - Données écologiques sur l'hibernation contrôlée des reines de Bourdons (Hym., Apoïdea, Bombinae, Bombus Latr.). Apidologie, I, 73-95.

Raw A., 1972. - The biology of the solitary bee Osmia rufa L. (Megachilidae) - Trans. R. entomol. Soc. Lond. 124, 213-229.

TAsei J. N., 1972. - Observations préliminaires sur la biologie d'Osmia coerulescens L. pollinisateur de la luzerne. Apidologie 3, 149-165.

TASEI J. N., sous presse. - Le comportement de nidification chez Osmia cornuta Latr. et Osmia rufa L. (Hym. Megachilidae) Apidologie. 